Victoor, A., Rademakers, J., Reitsma-van Rooijen, M., Jong, J. de, Delnoij, D., Friele, R. The effect of the proximity of patients' nearest alternative hospital on their intention to search for information on hospital quality. Journal of Health Services Research \& Policy: 2014, 19(1) 4=-11 nivel

\begin{tabular}{|l|l|}
$\begin{array}{l}\text { Postprint } \\
\text { Version }\end{array}$ & 1.0 \\
\hline Journal website & http://hsr.sagepub.com/content/19/1/4.short \\
\hline Pubmed link & $\underline{\text { http://www.ncbi.nlm.nih.gov/pubmed/23945678 }}$ \\
\hline DOI & $10.1177 / 1355819613498380$ \\
\hline
\end{tabular}

This is a NIVEL certified Post Print, more info at http://www.nivel.eu

\title{
The effect of the proximity of patients' nearest alternative hospital on their intention to search for information on hospital quality
}

\author{
AAFKE VICTOOR ${ }^{1,2} \Uparrow$, JANY RADEMAKERS ${ }^{1}$, MARGREET REITSMA-VAN ROOIJEN ${ }^{1}$, JUDITH DE \\ JONG $^{1}$, DIANA DELNOIJ ${ }^{2,3}$, ROLAND FRIELE ${ }^{1,2}$ \\ ${ }^{1}$ NIVEL, Netherlands Institute for Health Services Research, Utrecht, the Netherlands \\ ${ }^{2}$ Tilburg School of Social and Behavioural Sciences, Tilburg University, Tranzo, the \\ Netherlands \\ ${ }^{3}$ Health Care Insurance Board (CVZ), Quality Institute, Diemen, the Netherlands
}

\begin{abstract}
Objective In several countries, patients are encouraged to choose health care providers. Simultaneously, there is a tendency towards the concentration of health care, which might lessen the choice. Our aim was to assess the impact of patients' distances from their providers on their information-seeking, which is one element of choice.

Methods Two thousand members of the Dutch Health Care Consumer Panel were sent a questionnaire that included a question on whether they would search for information about hospitals to inform their choice. Distances from providers were obtained from a database that includes the distances between all postcodes in the Netherlands. To assess the influence of distance on information-seeking, logistic regression analyses were conducted.
\end{abstract}

Results There was a good response (75\%). Older, less educated respondents were less inclined to seek information when their nearest alternative was located further away (OR 0.85; 95\% CI: 0.79-0.92), and younger, more educated respondents were more inclined to search in this situation (OR 1.11; 95\% CI: 1.01-1.22).

Conclusions As fewer older, less educated patients would search for information to guide their choice, they might not opt for the best hospital. Additionally, the need for providers to compete for the patronage of these patients might be lessened. 
Victoor, A., Rademakers, J., Reitsma-van Rooijen, M., Jong, J. de, Delnoij, D., Friele, R. The effect of the proximity of patients' nearest alternative hospital on their intention to search for information on hospital quality. Journal of Health Services Research \& Policy: 2014, 19(1) 4 -117

\section{INTRODUCTION}

Patients' choice of health care provider has become a prominent policy issue in several countries as one element of regulated competition. ${ }^{1}$ Patients are encouraged to make an active choice or, in other words, to invest effort in acquiring information about price and quality of providers and to choose between them based on that information. It is anticipated that this will prompt providers to compete for patients by optimizing the care they deliver. ${ }^{1,2}$ Various measures have been taken to encourage patients to choose, such as publishing information on the quality of care. ${ }^{1}$ However, concentrating care at fewer locations and encouraging health care insurers to selectively contract care may limit the choice.,

Although patients are expected to choose, they generally opt for the default provider, often the nearest one. ${ }^{5}$ Whether patients choose varies according to provider, patient and health care system characteristics. ${ }^{6 \Downarrow \Downarrow \Downarrow^{-10}}$ A key factor seems to be whether patients have alternatives to their nearest provider. Because patients are averse to travel for health care, ${ }^{5}$ the distance to a provider might influence whether they consider it an alternative. When the nearest alternative is too far away, patients may not feel they have a choice. Whether providers are deemed viable may depend on their absolute distance from a patient's home or their distance relative to a patient's closest provider. A provider located $35 \mathrm{~km}$ away may not be seen as an alternative by somebody whose nearest provider is $5 \mathrm{~km}$ away while it might seem viable to somebody whose nearest provider is $32 \mathrm{~km}$ away.

A few studies have been carried out on the effect of the distance of alternative providers on choice. For instance, a study investigating why patients bypass the nearest hospital found that the relative distance to an alternative provider negatively influences bypassing. ${ }^{7}$ Another study found that patients are more likely to bypass nearby providers in areas with a high concentration of providers. ${ }^{11}$ Furthermore, whether patients consider a particular hospital to be an alternative depends on its distance from their preferred hospital. ${ }^{12}$ However, to our knowledge, the effect of the absolute distance to the nearest alternative on the choice behavior of various patient groups has never been researched or compared to the relative distance. The current work also has societal value. Patients are encouraged to choose, while their options are being restricted at the same time. If this leads to fewer choices, it might affect the functioning of patient choice as an instrument to enhance competition.

Searching for information on providers is an important element of making an active choice. ${ }^{2}$ Our aim was to investigate whether patients in the Netherlands would search for information to choose a hospital and what impact the proximity of their nearest alternative hospital has on whether they would undertake a search or not. The proximity of patients' nearest alternative hospital is operationalized as the absolute distance to their nearest alternative hospital and the distance to this hospital relative to the distance to their nearest one. We focus on the choice of a hospital because hospital care is an aspect of health care where alternatives and opportunities to choose are available. In the Dutch Health Care system, patient choice is encouraged, there is a relatively large number of hospital beds (4.7 beds per 1,000 population), and having health care insurance, which covers the hospital costs incurred by patients, is mandatory. ${ }^{13,14}$

We hypothesized that patients who live further away from their second nearest hospital are less prone to choose actively. ${ }^{7}$ The following patient groups are also less prone to make an active choice: older, low educated, ${ }^{5}$ male, ${ }^{10}$ less healthy patients ${ }^{9}$, 
Victoor, A., Rademakers, J., Reitsma-van Rooijen, M., Jong, J. de, Delnoij, D., Friele, R. The effect of the proximity of patients' nearest alternative hospital on their intention to search for information on hospital quality. Journal of Health Services Research \& Policy: 2014, 19(1) 4 -117

patients with chronic diseases ${ }^{8}$, and patients whose nearest hospital delivers top clinical care or is not a teaching hospital ${ }^{7}$. Therefore, we expect them to be less inclined to search for information. Additionally, distance has a larger negative influence on information seeking for older, less highly educated, less healthy patients and patients with chronic diseases. These groups are less mobile $\mathrm{e}^{13,14}$ and if the nearest alternative hospital is located too far away, they might not consider it at all.

\section{METHODS}

\section{Participants}

In March 2011, a questionnaire was sent to a sample from the Dutch Health Care Consumer Panel that was representative for the Dutch population in sex and age (18 years and above) $(\mathrm{N}=2000)$. The panel is managed by the Netherlands Institute for Health Services Research and registered with the Dutch Data Protection Authority. ${ }^{17}$ Migrants and less educated people are under-represented in the panel. As per their previously stated preferences, half the sample $(\mathrm{N}=928)$ received a written questionnaire while the other half $(\mathrm{N}=1072)$ received an online questionnaire.

\section{Instruments}

The questionnaire included 52 questions regarding a large variety of health topics. It included the following question: 'If you are referred to a hospital/specialist, would you look for information to help you to choose one?' Other questions were not relevant for this study. Up-to-date information was available about the backgrounds of all panel members, including their age and residential four-digit postcode.

Data on the hospitals were collected from three websites. ${ }^{18 \Downarrow-20}$ We included general hospitals, teaching hospitals and outpatient departments. Hospitals specializing in a few specific conditions, independent treatment facilities and private clinics (typically devoted to a few specific conditions) were not included. Because respondents were asked a question that was not focused on a specific condition, we expected them to consider only general hospitals offering a wide range of care.

\section{Analysis}

The absolute distances were defined as the shortest route by car $(\mathrm{km})$ between patients' residential postcodes and the postcode of their second nearest hospital. These routes were obtained from a database that includes the distances between all four-digit postcodes in the Netherlands. ${ }^{21}$ For the relative distance, we assumed that people perceive a difference between two values differently depending on the size of the baseline value. ${ }^{22}$ Therefore, we defined the relative distance as a ratio, i.e. the distance to the alternative divided by the distance to the nearest hospital.

We treated a hospital with several sites as a single hospital and calculated the distance to the site closest to a patient's home (Box 1). We divided both distance variables into 10 groups of an equal number of observations, since the data were positively skewed.

\section{[Box 1]}

Calculation of the average distance between two addresses in one postcode. Statistical analysis of the data was performed using Stata statistical software. Frequencies were calculated for background characteristics of the respondents and hospitals, and the question of whether patients would search for information. We 
Victoor, A., Rademakers, J., Reitsma-van Rooijen, M., Jong, J. de, Delnoij, D., Friele, R. The effect of the proximity of patients' nearest alternative hospital on their intention to search for information on hospital quality. Journal of Health Services Research \& Policy: 2014, 19(1) 4 -11

weighed frequencies for this question for age (18-39, 40-64, 64 and above) and sex, so that these results could be generalized to the Dutch population, based on composition data for the Dutch population on 1 January $2011^{24}$

To assess the influence of distance to the nearest alternative hospital on information seeking, we first conducted univariate logistic regression analyses on the influence of distance on information-seeking. Next, we built a multiple logistic regression model by means of a manually executed forward strategy. The null model only included the distance to the nearest alternative. Variables were considered for inclusion in the model when bivariate logistic regression on the influence of the variable and distance on information seeking yielded a p value lower than 0.25 . Since we divided both distance variables into 10 groups, every one-unit increase in the distance to the nearest alternative means 'every transition to a successive $10 \%$ group'.

\section{RESULTS}

A total of 1500 members completed the questionnaire (75\%). Table 1 shows the background characteristics of the participants and hospitals. Of the respondents, 609 (41.2\%) indicated that they would look for information to help them to choose a hospital/specialist. Whether they would search for information is significantly influenced by the proximity of the nearest alternative hospital. Every one-unit increase in the absolute distance to the nearest alternative leads to a $4 \%(p=0.018)$ decrease in the odds to search for information.

\section{[TABLE 1]}

Background characteristics of the participants and the hospitals.

Table 2 shows the results of the bivariate analyses. Because only the absolute distance to the second nearest hospital has a significant influence on whether respondents search for information, the relative distance was not included in the models. Interaction terms were only reported when significant. The absolute distance to the nearest alternative hospital negatively influences information seeking. Additionally, the odds for women to search for information are $66 \%$ higher than the odds for men, and the odds for respondents with chronic diseases to search for information are 37\% lower than the odds for respondents without. The effect of the distance to the nearest alternative provider on information seeking varies for different patient groups. For respondents aged 65 or above, every one-unit increase in the absolute distance to the nearest alternative leads to a $12 \%$ decrease in the odds to search for information, and for less highly educated respondents, every one-unit increase leads to a 7\% decrease (Figure 1).

\section{[TABLE 2]}

Bivariate logistic regression analyses testing the effects of distance and respondent and nearest hospital characteristics on whether respondents would search for information.

\section{[FIGURE 1]}

The effect of the interaction of age and education level with the absolute distance to the nearest alternative hospital on information-seeking behavior. 
Victoor, A., Rademakers, J., Reitsma-van Rooijen, M., Jong, J. de, Delnoij, D., Friele, R. The effect of the proximity of patients' nearest alternative hospital on their intention to search for information on hospital quality. Journal of Health Services Research \& Policy: 2014, 19(1) 4 -117

Table 3 shows the final multiple logistic regression model. Similar results were found, but the effect of distance had to be calculated for four different groups. For younger, more highly educated respondents, every one-unit increase in the distance to the nearest alternative leads to an $11 \%$ increase in the odds to search for information, while for older and less educated respondents, every one-unit increase leads to a $15 \%$ decrease (Figure 2 ).

[TABLE 3]

Final multiple logistic regression model that explains information seeking $(\mathrm{N}=1145)$.

\section{[FIGURE 2]}

The effect of the combined interaction of age and education level with the absolute distance to the nearest alternative hospital on information-seeking behavior.

\section{Discussion}

The majority of patients would not search for comparative information about hospitals/specialists when they need hospital care. Whether they search for information is influenced by the availability of a realistic alternative in terms of its absolute proximity. However, this influence is not the same for everyone. Older, less educated patients are less likely to search for information when they live further away from their nearest alternative hospital. Surprisingly, younger more highly educated patients are more likely to search for information in this situation. Furthermore, people with long-term conditions are less likely to search for information and women are more likely to.

Although most of our findings are in line with our hypotheses, there are three deviations from existing literature. First, the group stating an intention to search for information is quite large compared to the low uptake of patient choice of health care providers found in previous research. ${ }^{5,25}$ This may result from the fact that people generally intend to choose actively, whereas ultimately they do not. ${ }^{26}$

Second, contrary to existing literature, ${ }^{7}$ we discovered that the absolute and not the relative distance to people's nearest alternative hospital influences information seeking. This may be caused by our operationalizations of active, informed choice (i.e. future information seeking to guide provider choices as opposed to a previous choice) and of travel costs (distance in kilometres as opposed to travel time). Finally, unlike previous studies, ${ }^{7,9}$ we did not find any effect of hospital type and general health on information-seeking. Existing literature, however, only studied the influence of hospital type on choice, whereas we investigated its additional effect while adjusting for distance.

This study has several implications. Fewer less-educated older patients may make active choices because of the limited options in their proximity due to the concentration and selective contracting of health care. Consequently, they might not opt for the best hospital. Additionally, since the elderly comprise the majority of patients, the need for providers to compete on quality and price to obtain patients might be reduced. In the end, the concentration and selective contracting of health care is at odds with patient choice, while both are advocated as a means to improve health care efficiency, either directly or by stimulating competition. Health care 
Victoor, A., Rademakers, J., Reitsma-van Rooijen, M., Jong, J. de, Delnoij, D., Friele, R. The effect of the proximity of patients' nearest alternative hospital on their intention to search for information on hospital quality. Journal of Health Services Research \& Policy: 2014, 19(1) 4 -117

policy should therefore choose between empowering either patients or insurers and providers as a mechanism to improve health care quality.

The strengths of this study are that it tests the effect of both the relative distance and the absolute distance to patients' nearest alternative hospital on information seeking. In addition, we took into account that people may perceive the extra distance to their nearest alternative provider differently depending on the distance to their nearest provider. One limitation concerns the adjustment of the distances between respondents and hospitals, because researchers are not allowed to use all six characters of patients' postcodes. Another limitation is that we asked respondents a hypothetical question. Despite this, we found an influence of the proximity of people's nearest alternative hospital on information-seeking. Finally, the question that was posed to the respondents was not narrowed to a specific condition. It would be interesting to study the effect of the proximity of patients' nearest alternative on choice with respect to specific health care conditions, thereby including treatment facilities specialized in only those conditions in the analyses.

Further research should also investigate the effect of the proximity of patients' nearest alternative on choice in other countries. The Netherlands is unusual because of several factors related to its health care system. Additionally, different operationalizations of active choice and travel cost need to be studied.

\section{FUNDING}

This work was supported by the Dutch Ministry of Education, Culture and Science (grant number BEK/BPR-2009/24966 U).

\section{ACKNOWLEDGEMENTS}

We thank all respondents who filled out our questionnaire. We also thank Mike Wilkinson for copyediting the work.

\section{REFERENCES}

4 lkkersheim DE, Koolman X Dutch healthcare reform: did it result in better patient experiences in hospitals? A comparison of the consumer quality index over time. BMC Health Serv Res2012; 12: 1-6.

Lako Ch J, Rosenau P Demand-driven care and hospital choice. Dutch health policy toward demand-driven care: results from a survey into hospital choice. Health Care Anal2009; 17: 20-35.

$\triangleleft$ Boonen LHHM. Consumer channeling in health care: (im)possible? PhD Thesis. Erasmus Universiteit Rotterdam, Rotterdam, 2009.

$\triangleleft$ Glanville J, Duffy S, Mahon J, et al. Impact of hospital treatment volumes on patient outcomes. York: York Health Economics Consortium, University of York, 2010, pp.1-21.

$\triangleleft$ Victoor A, Delnoij DMJ, Friele RD, et al Determinants of patient choice of healthcare providers: a scoping review. BMC Health Serv Res2012; 12: 1-16.

«Tai WT, Porell FW, Adams EK Hospital choice of rural Medicare beneficiaries: patient, hospital attributes, and the patient-physician relationship. BMC Health Serv Res2004; 3966 Pt 1): 1903-1922.

$\checkmark$ Varkevisser M, van der Geest SA Why do patients bypass the nearest hospital? An empirical analysis for orthopaedic care and neurosurgery in the Netherlands. Eur J Health Econ2007; 8: 287-295.

$\triangleleft$ Dealey C The factors that influence patients' choice of hospital and treatment. Br J Nurs2005; 14: 576-579. 
Victoor, A., Rademakers, J., Reitsma-van Rooijen, M., Jong, J. de, Delnoij, D., Friele, R. The effect of the proximity of patients' nearest alternative hospital on their intention to search for information on hospital quality. Journal of Health Services Research \& Policy: 2014, 19(1) 4-117

$\triangleleft$ Harris KM How do patients choose physicians? Evidence from a national survey of enrollees in employment-related health plans. Health Serv Res2003; 38: 711-732.

$\triangleleft$ Robertson R, Burge P The impact of patient choice of provider on equity: analysis of a patient survey. J Health Serv Res Policy2011; 16(Suppl 1): 22-28.

$\triangleleft$ Hyndman JCG, Holman CDJ, Pritchard DA The influence of attractiveness factors and distance to general practice surgeries by level of social disadvantage and global access in Perth, Western Australia. Soc Sci Med2003; 56: 387-403.

$\triangleleft$ Varkevisser M, van der Geest S and Schut F. Quality competition in regulated hospital markets: consumer information and patient choice for angioplasty. Patient choice, competition and antitrust enforcement in Dutch hospital markets. PhD Thesis. Rotterdam: Erasmus Universiteit Rotterdam, 2009, pp.117-149.

-Enthoven A, van de Ven W Going Dutch-managed-competition health insurance in the Netherlands. N Engl J Med2007; 357: 2421-2423.

$4 O E C D$. Health at a Glance: Europe 2012. Paris: OECD Publishing, 2012.Butler AA, Menant JC, Tiedemann AC, et al Age and gender differences in seven tests of functional mobility. J NeuroEng Rehabil2009; 6: 1-9.Sainio P, Martelin T, Koskinen S, et al Educational differences in mobility: the contribution of physical workload, obesity, smoking and chronic conditions. J Epidemiol Commun H2007; 61: 401-408.

$\triangleleft$ Brabers AEM, Reitsma-van Rooijen M and de Jong JD. Consumentenpanel Gezondheidszorg. Basisrapport met informatie over het panel (2011). Utrecht: NIVEL, 2011, pp.1-58.

«RIVM. Kiesbeter.nl. http://www.kiesbeter.nl/zorgverleners/ (accessed August 2012). $\triangleleft$ BSL, NPCF. ZorgkaartNederland. www.ZorgkaartNederland.nl (accessed August 2012). $\triangleleft$ Medical Media. Ziekenhuis.nl. www.ziekenhuis.nl (accessed August 2012).

$\checkmark$ Geodan IT b.v. Drive Time Matrix van Nederland. Product documentatie. Amsterdam: Geodan IT b.v. 2012, pp.1-7.

4 Blake R, Sekuler R Appendix. Behavioral methods for studying perception. Perception, New York: The McGraw-Hill Companies, 2006, pp. 553-568.

$\triangleleft$ CBS. Bevolking; geslacht, leeftijd en burgerlijke staat, 1 Januari 2012. www.cbs.nl (accessed August 2012).

$\triangleleft$ Dijs-Elsinga J, Otten W, Versluijs M, et al Choosing a hospital for surgery: the importance of information on quality of care. Med Decis Making2010; 30: 544-555. «Schwartz L, Woloshin S, Birkmeyer J How do elderly patients decide where to go for major surgery? Telephone interview survey. BMJ2005; 331: 821-827.

TABLES, BOXES AND FIGURES 
Box 1. Calculation of the average distance between two addresses in one postcode.

When a respondent's postcode was the same as that of their nearest hospital, the distance was set at the average distance between two addresses in one postcode: $1.1 \mathrm{~km}$. The average distance of an address (a six-digit postcode) to the centre of its four-digit postcode is $800 \mathrm{~m}$. ${ }^{21}$ If we assume this area to be circular in shape, its radius ( $r$ ) must be 1.5 times the average distance of an address to the centre of its postcode. The average distance between two points within an area is $(128 / 45 \pi)^{*}$ r. Given that the average distance of an address to the centre of its postcode is $800 \mathrm{~m}$, this gives $1.5^{*} 128 / 45 \pi^{*} 800=1086 \mathrm{~m}$, rounded up to $1100 \mathrm{~m}^{23}$ When the distance to the nearest alternative hospital was between zero and $1.1 \mathrm{~km}$, this distance was used instead (applied to two hospitals). 
Victoor, A., Rademakers, J., Reitsma-van Rooijen, M., Jong, J. de, Delnoij, D., Friele, R. The effect of the proximity of patients' nearest alternative hospital on their intention to search for information on hospital quality. Journal of Health Services Research \& Policy: 2014, 19(1) 4-117

Table I. Background characteristics of the participants and the hospitals.

\begin{tabular}{|c|c|c|c|}
\hline Variable & $N(\%)$ & Md $(\mathrm{IQR})^{2}$ & Range \\
\hline \multicolumn{4}{|l|}{ Distance } \\
\hline Distance to hospital $\mathrm{I}^{\mathrm{b}}(\mathrm{km})$ & $1396(100.0)$ & $4.4(6.3)$ & $0.6-37.9$ \\
\hline Distance to hospital $2^{c}(\mathrm{~km})$ & $1396(100.0)$ & $15 . \mid(12.72)$ & $0.8-68.5$ \\
\hline \multicolumn{4}{|l|}{ Respondent characteristics } \\
\hline Age & $1500(100.0)$ & & \\
\hline Low (18-39 years) & $339(22.6)$ & & \\
\hline Middle (40-64 years) & $627(41.8)$ & & \\
\hline High (>64 years) & $534(35.6)$ & & \\
\hline Gender & $1500(100.0)$ & & \\
\hline Women & $810(54.0)$ & & \\
\hline Education level & $1447(96.5)$ & & \\
\hline Low $^{d}$ & $237(16.4)$ & & \\
\hline Medium ${ }^{e}$ & $865(59.8)$ & & \\
\hline $\mathrm{High}^{\prime}$ & $345(23.8)$ & & \\
\hline Ethnicity & $1496(99.7)$ & & \\
\hline Dutch & $|40|(93.6)$ & & \\
\hline Income & $1412(94.1)$ & & \\
\hline Low $(<=1750)$ & $428(30.3)$ & & \\
\hline Middle (1750-2500) & $443(3 \mid .4)$ & & \\
\hline High $(>=2500)$ & $541(38.3)$ & & \\
\hline Subjective general physical health & I476 (98.4) & & \\
\hline Medium/ bad & $244(16.5)$ & & \\
\hline Good & $873(59.2)$ & & \\
\hline Very good/ excellent & $359(24.3)$ & & \\
\hline Chronic or long-term disease & $1237(82.5)$ & & \\
\hline Yes & $917(74.1)$ & & \\
\hline \multicolumn{4}{|l|}{ Hospital characteristics } \\
\hline Hospital type & $201(100.0)$ & & \\
\hline General hospital & $128(63.7)$ & & \\
\hline Teaching hospital & $8(4.0)$ & & \\
\hline Outpatient clinic & $65(32.3)$ & & \\
\hline Top-clinical care & $201(100.0)$ & & \\
\hline Delivers top-clinical care & $62(30.8)$ & & \\
\hline
\end{tabular}

${ }^{2}$ Median (interquartile range).

'Hospital $\mathrm{I}=$ nearest hospital.

${ }^{\complement}$ Hospital 2 = nearest alternative hospital.

¿Low = primary school or only vocational training.

'Medium = secondary school or intermediate vocational training.

'High $=$ tertiary education. 
Victoor, A., Rademakers, J., Reitsma-van Rooijen, M., Jong, J. de, Delnoij, D., Friele, R. The effect of the proximity of patients' nearest alternative hospital on their intention to search for information on hospital quality. Journal of Health Services Research \& Policy: 2014, 19(1) 4-11 7

Table 2. Bivariate logistic regression analyses testing the effects of distance and respondent and nearest hospital characteristics on whether respondents would search for information.

\begin{tabular}{|c|c|c|c|}
\hline Variable & Odds & $\mathrm{p}$ & $95 \% \mathrm{Cl}$ \\
\hline \multicolumn{4}{|c|}{ Distance to the nearest alternative hospital } \\
\hline Absolute distance $(\mathrm{N}=\mid 379)$ & $0.96 * * *$ & 0.018 & $0.92-0.99$ \\
\hline Relative distance $(N=1379)$ & 1.00 & 0.961 & $0.96-1.04$ \\
\hline \multicolumn{4}{|l|}{ Respondent characteristics } \\
\hline \multicolumn{4}{|l|}{ Age $(N=\mid 379)$} \\
\hline Low/middle (I8-64) & Reference & Reference & Reference \\
\hline High $(>64)$ & 0.94 & 0.784 & $0.62-1.43$ \\
\hline Distance & 0.99 & 0.611 & $0.94-1.03$ \\
\hline Low/middle*distance & Reference & Reference & Reference \\
\hline High*distance & $0.89 * *$ & 0.005 & $0.82-0.97$ \\
\hline \multicolumn{4}{|l|}{ Gender $(N=1379)^{2}$} \\
\hline Men & Reference & Reference & Reference \\
\hline Women & $1.66 *$ & 0.000 & $1.34-2.07$ \\
\hline Distance & $0.96^{\text {tw1* }}$ & 0.028 & $0.92-1.00$ \\
\hline \multicolumn{4}{|l|}{ Education level $(\mathrm{N}=1343)$} \\
\hline Low $^{b} /$ medium ${ }^{c}$ & 0.91 & 0.685 & $0.58-1.43$ \\
\hline High $^{d}$ & Reference & Reference & Reference \\
\hline Distance & 1.06 & 0.158 & $0.98-1.14$ \\
\hline Low/medium*distance & $0.88^{* *}$ & 0.006 & $0.81-0.96$ \\
\hline High"distance & Reference & Reference & Reference \\
\hline \multicolumn{4}{|c|}{ Subjective general physical health $(\mathrm{N}=1368)^{\mathrm{a}}$} \\
\hline Medium/bad & Reference & Reference & Reference \\
\hline Good & 0.95 & 0.715 & $0.699-1.28$ \\
\hline Very good/excellent & 1.17 & 0.363 & $0.83-1.66$ \\
\hline Distance & $0.96 \cdots$ & 0.024 & $0.92-0.99$ \\
\hline \multicolumn{4}{|c|}{ Chronic or long-term disease $(\mathrm{N}=1178)^{\mathrm{a}}$} \\
\hline No & Reference & Reference & Reference \\
\hline Yes & $0.63^{\text {*ok }}$ & 0.001 & $0.48-0.82$ \\
\hline Distance & $0.95^{\text {*ank }}$ & 0.014 & $0.91-0.99$ \\
\hline \multicolumn{4}{|l|}{ Nearest hospital characteristics } \\
\hline \multicolumn{4}{|l|}{ Hospital type $(\mathrm{N}=1379)^{\mathrm{a}}$} \\
\hline General hospital & Reference & Reference & Reference \\
\hline Teaching hospital & 1.58 & 0.075 & $0.95-2.63$ \\
\hline Outpatient clinic & 1.09 & 0.529 & $0.83-1.42$ \\
\hline Distance & $0.96^{* * 2 * k}$ & 0.031 & $0.92-1.00$ \\
\hline \multicolumn{4}{|c|}{ Delivers top-clinical care $(\mathrm{N}=1379)^{2}$} \\
\hline No top-clinical care & Reference & Reference & Reference \\
\hline Top-clinical care & 1.06 & 0.636 & $0.84-1.33$ \\
\hline Distance & $0.96^{* * 6 *}$ & 0.029 & $0.92-1.00$ \\
\hline
\end{tabular}

${ }^{*} p<0.001 ;{ }^{* * p}<0.01 ; *{ }^{*}<0.05 ; \mathrm{NA}=$ not applicable; distance $=$ absolute distance to the nearest alternative hospital.

"The results for the model without the interaction term are reported, because no interaction was found between this variable and distance.

bow = primary school or only vocational training.

${ }^{c}$ Medium $=$ secondary school or intermediate vocational training.

${ }^{\mathrm{d}} \mathrm{High}=$ terthary education. 
Victoor, A., Rademakers, J., Reitsma-van Rooijen, M., Jong, J. de, Delnoij, D., Friele, R. The effect of the proximity of patients' nearest alternative hospital on their intention to search for information on hospital quality. Journal of Health Services Research \& Policy: 2014, 19(1)_4-117

\section{FIGURE 1}

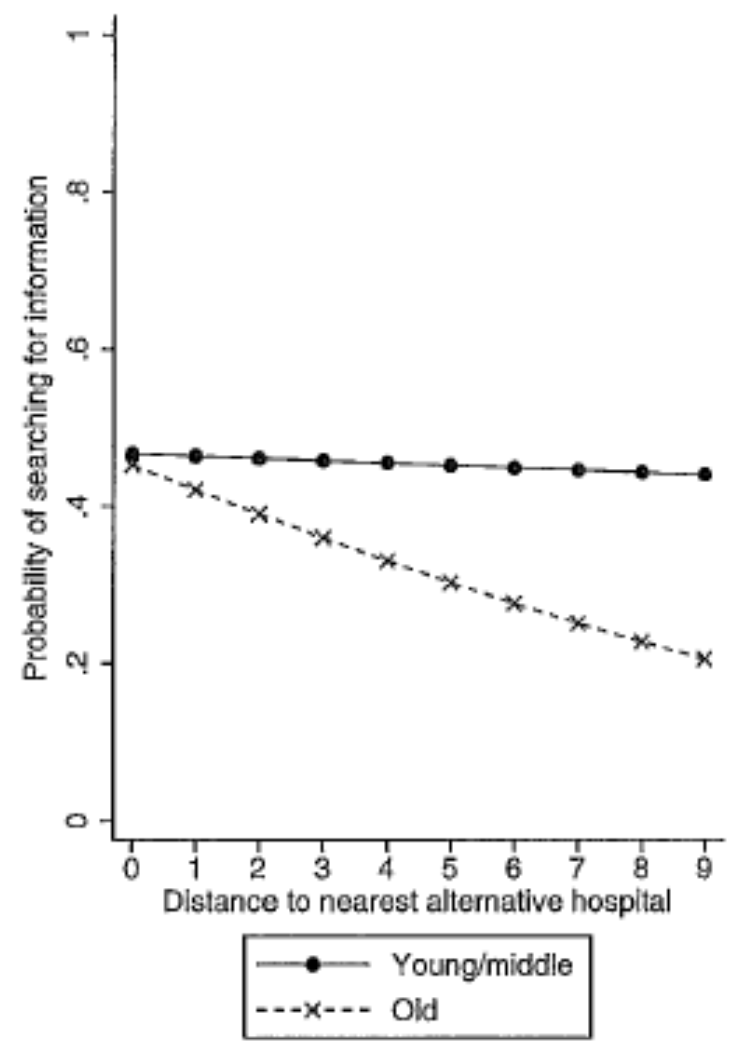

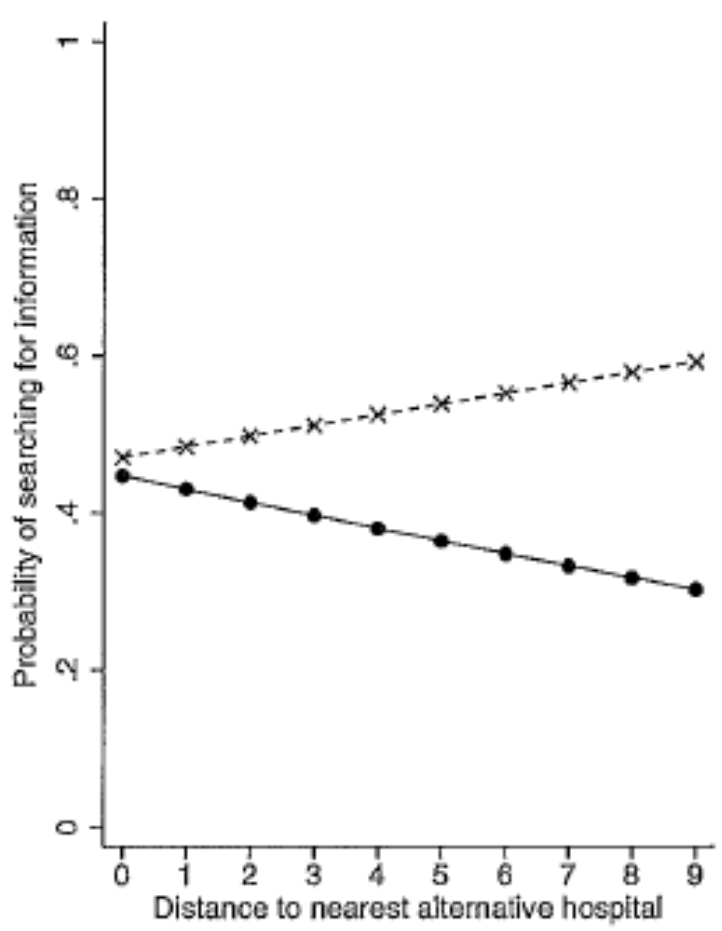

$\longrightarrow$ Low/medium education 
Victoor, A., Rademakers, J., Reitsma-van Rooijen, M., Jong, J. de, Delnoij, D., Friele, R. The effect of the proximity of patients' nearest alternative hospital on their intention to search for information on hospital quality. Journal of Health Services Research \& Policy: 2014, 19(1)_4-117

nivel

Table 3. Final multiple logistic regression model that explains information seeking ( $N=1 \mid 45)$.

\begin{tabular}{|c|c|c|c|}
\hline Variable & Odds & $\mathrm{P}$ & $95 \% \mathrm{Cl}$ \\
\hline Distance (absolute) & $1.11^{\text {*wo\% }}$ & 0.033 & $1.01-1.22$ \\
\hline \multicolumn{4}{|l|}{ Age } \\
\hline Low/middle (18-64 years) & Reference & Reference & Reference \\
\hline High (>64 years) & 1.15 & 0.547 & $0.73-1.80$ \\
\hline \multicolumn{4}{|l|}{ Gender } \\
\hline Men & Reference & Reference & Reference \\
\hline Women & $1.39 \%$ & 0.016 & $1.06-1.81$ \\
\hline \multicolumn{4}{|l|}{ Education level } \\
\hline Low $^{\mathrm{a}} /$ medium $^{\mathrm{b}}$ & 0.93 & 0.782 & $0.57-1.53$ \\
\hline $\mathrm{High}^{c}$ & Reference & Reference & Reference \\
\hline \multicolumn{4}{|l|}{ Chronic or long-term disease } \\
\hline No & Reference & Reference & Reference \\
\hline Yes & $0.68^{* * *}$ & 0.008 & $0.52-0.90$ \\
\hline \multicolumn{4}{|l|}{ Age*distance } \\
\hline Low/middle age ( $18-64$ years) & Reference & Reference & Reference \\
\hline High age (>64 years) & $0.88^{* * *}$ & 0.006 & $0.80-0.97$ \\
\hline \multicolumn{4}{|l|}{ Education level*distance } \\
\hline Low/medium education level & $0.88 * *$ & 0.009 & $0.79-0.97$ \\
\hline High education level & Reference & Reference & Reference \\
\hline
\end{tabular}

${ }^{*} p<0.001 ;{ }^{*} p<0.01 ; * * *_{p}<0.05 ;$ Distance $=$ absolute distance to the nearest alternative hospital.

"Low $=$ primary school or only vocational training.

'Medium = secondary school or intermediate vocational training.

${ }^{C} \mathrm{High}=$ tertiary education; Model characteristics: $L R$ chi $^{2}=74.03, \mathrm{P}=0.000$, Pseudo $\mathrm{R}^{2}=0.0480$. 
Victoor, A., Rademakers, J., Reitsma-van Rooijen, M., Jong, J. de, Delnoij, D., Friele, R. The effect of the proximity of patients' nearest alternative hospital on their intention to search for information on hospital quality. Journal of Health Services Research \& Policy: 2014, 19(1)_4-117

\section{FIGURE 2}

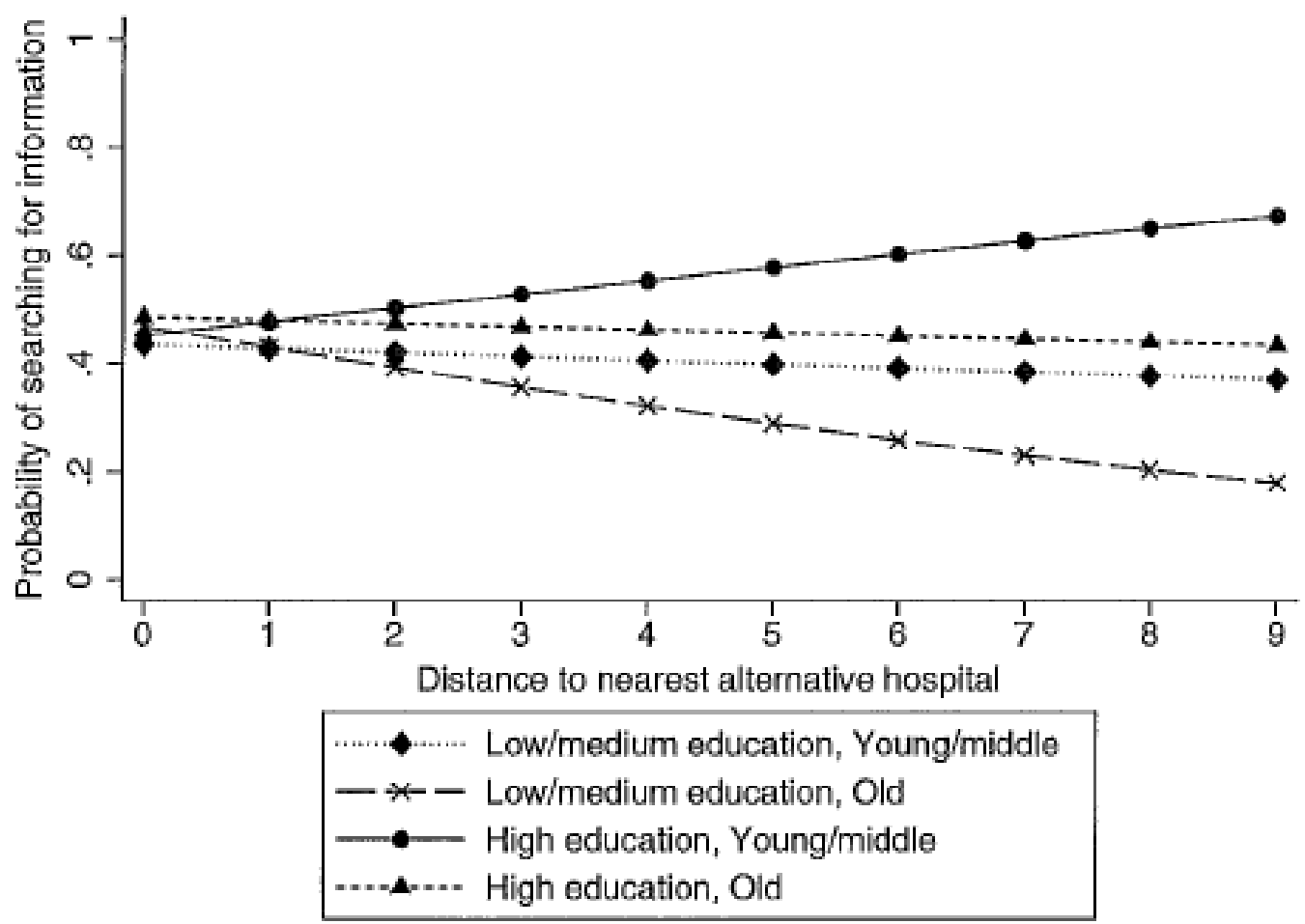

